\title{
Polarised Press Reporting about HIV Prevention: Social Representations of Pre-Exposure Prophylaxis (PrEP) in the UK Press
}

\author{
Rusi Jaspal \\ De Montfort University, Leicester, UK \\ Brigitte Nerlich \\ University of Nottingham
}

\begin{abstract}
Pre-exposure prophylaxis (PrEP) is a novel bio-medical HIV prevention option for individuals at high risk of HIV acquisition. Although PrEP has yielded encouraging results in various clinical trials, opponents argue that PrEP poses a number of risks to human health and to STI prevention efforts. Using qualitative thematic analysis and social representation theory, this article explores coverage of PrEP in the UK print media between 2008 and 2015 in order to map the emerging social representations of this novel HIV prevention strategy. The analysis revealed two competing social representations of PrEP: (1) as a positive development in the "battle" against HIV (the hope representation) and (2) as a medical, social and psychological setback in this battle, particularly for gay/bisexual men (the risk representation). These social representations map onto the themes of PrEP as a superlatively positive development; PrEP as a weapon in the battle against HIV/AIDS; and risk, uncertainty and fear in relation to PrEP. The hope representation focuses on taking (individual and collective) responsibility, while the risk representation focuses on attributing (individual and collective) blame. The implications for policy and practice are discussed.
\end{abstract}

\section{Keywords}

PrEP, HIV, HIV prevention, sexual health, media, social representations

\section{Citing this article}

Jaspal, R. \& Nerlich, B. (in press). Polarised Press Reporting about HIV Prevention: Social Representations of Pre-Exposure Prophylaxis (PrEP) in the UK Press. Health: An Interdisciplinary Journal for the Social Study of Health, Illness \& Medicine.

\section{Author biographies}

Rusi Jaspal is Professor of Psychology and Sexual Health and Deputy Director of the Mary Seacole Research Centre at De Montfort University Leicester, UK. He is Visiting Professor of Minority Research at Åbo Akademi University In Turku, Finland. He is a medical board member of NAZ: Sexual Health for Everyone, and a Fellow of the Royal Society for Public Health. Prof Jaspal has led many research projects related to the social psychological aspects of HIV/AIDS, several of which were funded by Public Health England.

Brigitte Nerlich is Professor of Science, Language and Society at the University of Nottingham. She has a D.Phil. in French linguistics and has been awarded a D.Litt. for her work on the social and political aspects of metaphor. Information about her work can be found at:

nottingham.academia.edu/BrigitteNerlich

\section{Contact details}

Professor Rusi Jaspal, Mary Seacole Research Centre, Faculty of Health \& Life Sciences, De Montfort University, Leicester LE1-9BH, UK. Tel: +44 (0)116 2507109 E-mail:

rusi.jaspal@cantab.net 


\section{Polarised Press Reporting about HIV Prevention: Social Representations of Pre-Exposure Prophylaxis (PrEP) in the UK Press}

Pre-exposure prophylaxis (PrEP) is a novel bio-medical approach for preventing HIV infection. The drug Truvada ${ }^{\circledR}{ }^{1}$ is currently used as PrEP in the US and in clinical trials in the UK. Several trials have demonstrated that, if taken correctly, PrEP can block a permanent infection from taking hold after HIV has entered the body. Yet, PrEP is not without its controversies - critics argue that PrEP poses a number of risks to human health and to STI prevention efforts (Lacor, 2012). While there has been some attitudinal research into PrEP (Brooks et al., 2012;

Tangmunkongvorakul et al., 2013), there has been no insight into social representations that are emerging in this debate. The print media can play an important role in setting the agenda for science and medicine, and can contribute to shaping public perceptions and policy debates. This article explores coverage of PrEP in the UK print media between 2008 (when PrEP was first mentioned in the UK press) and 2015 in order to map the emerging social representations of this novel HIV prevention strategy.

\section{HIV Prevention}

The Human Immunodeficiency Virus (HIV), which causes Acquired Immune Deficiency Syndrome (AIDS), has already claimed the lives of approximately 36 million people worldwide since the first clinical observations in 1981. It is estimated that in 201436.9 million people were living with HIV globally. ${ }^{2}$ In the UK, HIV prevalence is approximately $0.19 \%$ of the population aged between 15 and 59 - it is estimated that some 103,700 people are living with the virus, 17 per cent of whom are unaware of their positive serostatus (Public Health England, 2015). Men who have sex with men (MSM) suffer major inequalities in relation to HIV. According to Public Health England (2015), approximately 45,000 MSM were living with HIV in 2014, and in London it is estimated that 1 in 11 MSM did so. In 2014 there were 5850 new diagnosed cases of (sexually transmitted) HIV, of which 57\% were among MSM.

In the absence of a vaccine, prevention remains the most effective tool against HIV. Condom use has long been the preferred policy strategy in most developed countries. Although condoms remain a highly effective STI prevention method, it is clear that not everybody uses them consistently (Shernoff, 2006). In addition to condoms, other prevention techniques are used. For instance, MSM in particular may engage in 'serosorting', that is, they may have (condomless) sex with people who they believe share their HIV status (Golden et al., 2008). However, many HIV-positive individuals are unaware of their status and, due to the social stigma, some may choose not to disclose it. Furthermore, treatment as prevention constitutes an effective public health strategy, given that effective antiretroviral therapy (ART) suppresses the HIV-infected individual's viral load to 'undetectable' levels, thereby reducing their risk of onward transmission (Attia et al., 2009). Nevertheless, many new infections occur as a result of sexual intercourse with an HIV-infected individual who is not virally suppressed, either because they are unaware of their infection or because they have not yet started ART. In this context, rising HIV incidence has led to debates around novel prevention strategies.

\section{The Emergence of PrEP: Opportunities and Risks}

A series of clinical trials such as iPrEx (Grant et al., 2010), Partners PrEP (Baeten et al., 2010) and PROUD (McCormack et al., 2016), in which a subgroup of individuals are offered the experimental drug Truvada and a control group is offered a placebo drug, have repeatedly demonstrated the effectiveness of orally administered PrEP. In 2012 the US Food and Drug Administration approved the use of Truvada as PrEP among individuals at high risk of HIV acquisition, that is, those in a serodiscordant sexual relationship and those who do not use condoms consistently with partners of unknown serostatus. ${ }^{3}$ 
This decision has not met with unanimous acceptance. Michael Weinstein, president of the AIDS Healthcare Foundation, recently branded PrEP a "party drug" and warned of decreased condom use and increased STI incidence. ${ }^{4}$ Weinstein has since described PrEP as "an individual option, not a general solution". 5 Similarly, Richard Weinmayer, a research associate at the American Medical Association's Ethics Group, has claimed that PrEP will induce "sexual irresponsibility." "There is evidence of social stigma in relation to PrEP users who have been branded "Truvada whores" (Spieldenner, in press), which may induce shame and discourage use of PrEP. Most health insurance companies in the US at least partially fund PrEP when prescribed by a physician but there are some barriers to access (e.g. awareness and cost) (Bauermeister et al., 2013).

Uptake of PrEP has been relatively low (Flash et al., 2014), which may be attributed partly to the following concerns:

- PrEP is often positioned as a prevention technique that should be used alongside condoms. However, critics have voiced concerns that PrEP may encourage sexual risktaking and increase the incidence of other STIs. This reflects concerns regarding condom fatigue among MSM (Kalichman et al., 1998).

- Adherence to PrEP in the way prescribed is central to its effectiveness. Given that drug adherence has already been identified as a significant problem in treating HIV-positive patients (Fogarty et al., 2001), critics fear poor adherence to PrEP. Recent attitudinal research suggests that one's HIV-negative serostatus may create complacency vis-à-vis PrEP adherence (Tangmunkongvorakul et al., 2013). However, it is acknowledged that the IPERGAY clinical trial conducted in France and Canada demonstrated that intermittent dosing of PrEP (consisting of two Truvada pills taken 2-24 hours before a possible exposure, followed by a third pill 24 hours after the possible exposure and a fourth pill 48 hours after the possible exposure) is 86\% effective (Molina et al., 2015).

- The clinical trials have found no evidence of serious side effects of using PrEP, although minor side effects (e.g. stomach pains and loss of appetite) have been observed in some patients. PrEP sceptics fear potential long-term side effects (e.g. decreased kidney function).

- There is evidence of strains of HIV that are becoming resistant to tenofovir, one of the drugs in PrEP, which theoretically could compromise the effectiveness of PrEP (The TenoRes Study Group, 2016).

- HIV-positive individuals must never use PrEP because this could lead to future drug resistance, thereby limiting treatment options. Critics have highlighted their concern that some PrEP users may not have regular HIV tests and may become HIV-positive while using PrEP (inconsistently).

PrEP has not yet been approved in the UK. It has been available free of charge only to MSM enrolled in the PROUD study, which is the UK clinical trial of PrEP for use among MSM (McCormack et al., 2016). PrEP is not currently available on the UK National Health Service (NHS) but prominent UK HIV/AIDS organizations and sexual health charities, such as the Terrence Higgins Trust, National AIDS Trust, GMFA and NAZ: Sexual Health for Everyone have called for PrEP to be made available on the NHS. In March 2016, NHS England decided not to commission PrEP, arguing that commissioning HIV prevention services is the responsibility of local authorities. In response to this decision, supporters of PrEP have launched a petition calling for PrEP to be made available on the NHS. ${ }^{7}$ There are reports that some MSM are purchasing a generic version of PrEP Tenvir-EM (which contains the same drugs as Truvada) online, while others are engaging in "clinic hopping", that is, they falsely claim to have been at risk of HIV and are requesting post-exposure prophylaxis (PEP) ${ }^{8}$ at several sexual health clinics and using one of the drugs (Truvada) as PrEP. 


\section{Science and Media: The Role of Social Representations}

The media constitute a key source of societal information regarding scientific, technological and medical developments, like PrEP (Entman, 1989). When faced with a novel medical issue, the media tend to rely on metaphors and commonplace images in order to conceptualise and communicate about it. In this study, we draw upon Social Representations Theory (Moscovici, 1988), which focuses on collective elaborations of knowledge and how cultural meaning systems evolve. A social representation consists of a network of ideas, values and practices in relation to a given social object. Social representations enable us to understand the novel and unknown through two principal social psychological mechanisms - anchoring and objectification:

- Anchoring refers to the process of making something unfamiliar understandable by linking it to something familiar. For instance, by anchoring PrEP to the contraceptive pill, PrEP may be normalised and understood primarily in terms of the characteristics of the pill.

- Objectification is the process whereby unfamiliar and abstract objects are transformed into concrete and 'objective' common-sense realities - most notably through the use of metaphor - allowing us to map aspects of more familiar knowledge (the so-called source domain) onto more unfamiliar knowledge (the so-called target domain) (Lakoff and Johnson, 1980). For instance, the use of war metaphors can serve to construct PrEP as a powerful and effective weapon against HIV, thereby fostering positive expectations and hopes about its benefits, while metaphors describing PrEP as a 'party drug' taken recklessly may foster stigma, negative expectations and fear.

Although alternative theoretical frameworks like metaphor analysis and frame analysis were considered, we felt that a broad heuristic framework that could capture frames, metaphors and social imagery would be more appropriate. As a relatively novel and unknown medical development, PrEP has to be anchored to, and objectified in terms of existing constructs both in scientific and popular media. Therefore, Social Representations Theory provides an appropriate framework for examining the transition of PrEP from the world of science to the social psychological domain. This study stands in a long tradition of work focusing on infectious diseases from a social representations and media perspective from Susan Sontag's $(1978,1989)$ work on AIDS, its metaphors and social stigma to more recent work on infectious disease and society (Herek, 1990; Washer, 2010; Eicher \& Bangerter, 2015).

In this article we investigate this anchoring process in "real time" in order to observe emerging social representations of risk and benefits, hope and fear, blame and responsibility, which might influence both the production and uptake of PrEP. Furthermore, as Breakwell (2014) has argued, when social representations enter the social and psychological domain, individuals take a stance on them by "personalising" them. Although this exploratory study focuses on the print media only, it provides initial insights into the possible ways in which social representations of PrEP may become salient and relevant, and be accepted or rejected by people who could potentially benefit from PrEP.

We present a qualitative thematic analysis of the coverage of PrEP in the print media until May 2015 (when we conducted our analysis). We focus on the emerging social representations of PrEP during this period and scrutinise the contexts in which they appear and the functions that they may perform in debate and public understanding. More specifically, we examine whether PrEP is depicted positively or negatively, how it is anchored and objectified (e.g. which metaphors are used), and the policy responses that are concurrently legitimised.

However, it is important to acknowledge possible limitations to this approach. PrEP has not been a major issue of discussion in UK newspapers. This makes it an ideal case study for a small-scale qualitative analysis which attempts to trace the emerging contours of an important debate on HIV prevention. The print media constitute an important source of societal information regarding health and medicine and can at least set the tone for socio-political 
engagement with such issues, by influencing and reflecting policy agendas (Reese et al., 2003). Representations in the print media are often taken up and reproduced in other socio-political contexts like social media platforms. Our exploratory study excludes online discussions which are emerging quickly and which will thus need to be studied separately. There is, for example, a Facebook page on PrEP with almost 8500 subscribers, paid editorial staff, and a constant and lively discussion around the topic. The pharmaceutical industry too uses social media and, in the US, the Federal Drugs Administration (FDA) issues guidance on such engagement. ${ }^{9}$ Future studies of PrEP will need to monitor these developments, especially when studying the uptake of and debate around PrEP, in the UK, the US and elsewhere.

\section{METHOD}

\section{The Corpus}

Using the keywords "Pre-Exposure Prophylaxis" and "HIV" on the Nexis ${ }^{\circledR}$ news database, ${ }^{10}$ we conducted a search of all UK newspapers and extracted all articles published until 15/04/15 (our search date). This yielded a total of 59 newspaper articles, all of which were relevant and therefore included in the analysis. The first articles to mention PrEP were published in Metro (a daily newspaper distributed for free on public-transport services in UK urban centres) and London Lite (a now defunct daily newspaper that was distributed for free in Central London) on 20 November 2008 and the last appeared in The Independent on 9 April 2015. These outlets reflect a wide range of political perspectives and include both national and local newspapers.

Table 1 provides an overview of the distribution of articles included in the analysis:

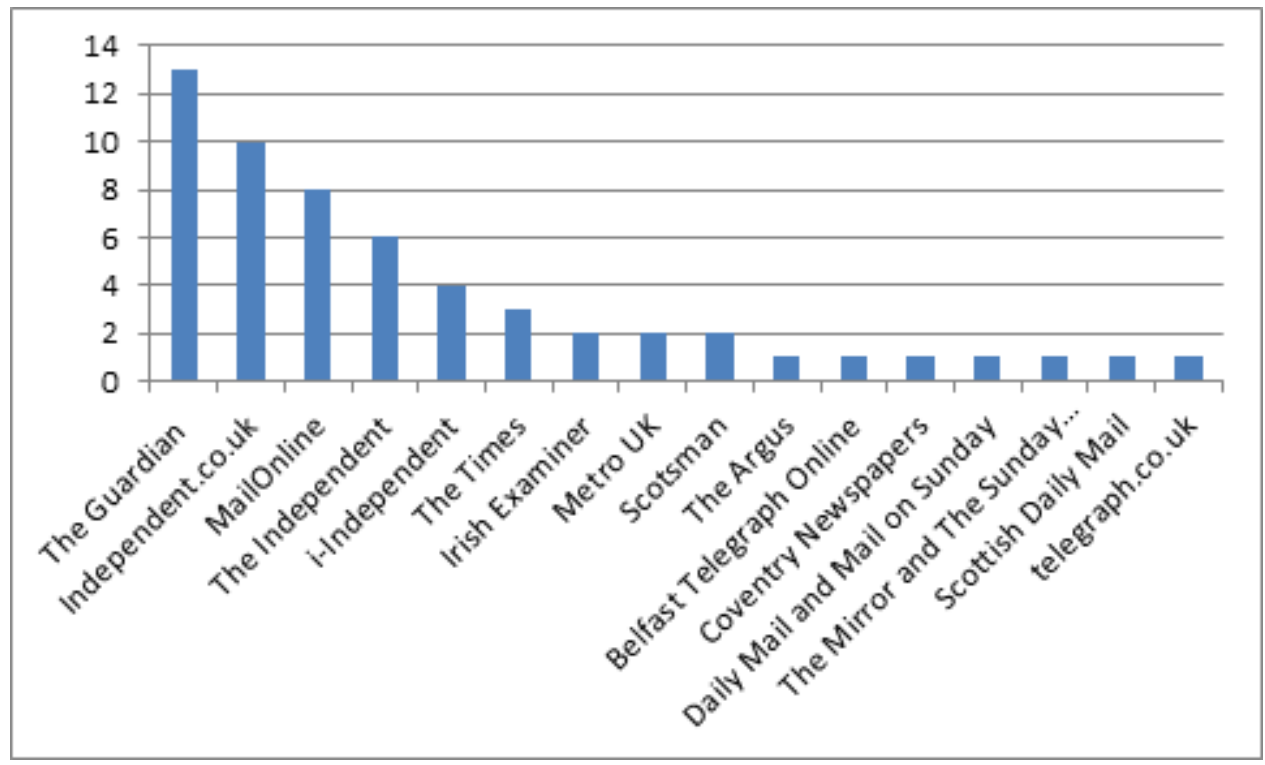

Table 1: Number of articles included in the analysis by outlet

\section{Analytical procedure}

We analysed the corpus using qualitative thematic analysis, which has been described as "a method for identifying, analysing and reporting patterns (themes) within data" (Braun \& Clarke, 2006 , p. 78). Thematic analysis allowed us to identify emerging social representations of PrEP as a socio-medical issue, and the dominant rhetorical strategies used to construct them.

We read and re-read the articles to familiarise ourselves with the broader themes that we subsequently discussed analytically. The left margin of each article was used to note initial observations that captured essential qualities, units of meaning and rhetorical techniques. We discussed our respective initial codes, which included inter alia general tone, particular forms of language, comparisons, categorisations and emerging patterns in the data. These initial codes 
were collated into preliminary themes, and arranged into a coherent structure that reflected the analysis. This process resulted in the identification of three superordinate themes.

In addition to describing dominant themes in the corpus, we identified linguistic elements, especially metaphors that performed the functions of anchoring and objectification. The superordinate themes can be considered social representations because they "assume a configuration where concepts and images can coexist without any attempt at uniformity, where uncertainty as well as misunderstandings are tolerated, so that discussion can go on and thoughts circulate" (Moscovici, 1988, p. 233). Thus, the hope and risk representations described in this article, collectively, constitute a "framework" for understanding and discussing potentially "divergent concepts, inconsistent ideas and paradoxical meanings" in relation to PrEP (Rose et al., 1995, p.4). In the analysis below, we provide extracts from the articles that exemplify the superordinate themes.

\section{ANALYSIS}

There were two competing social representations of PrEP: (1) as a positive development in the "battle" against HIV (the hope representation) and (2) as a medical, social and psychological setback in this "battle", particularly for gay/bisexual men (the risk representation). These social representations map onto the themes of PrEP as a superlatively positive development; PrEP as a weapon in the battle against HIV/AIDS; and risk, uncertainty and fear in relation to PrEP.

\section{The hope representation}

PrEP was constructed as a key development in HIV prevention in the articles examined. Truvada as PrEP was described metaphorically as a "revolution," "revolutionary," a "wonder drug," "game changer," and "the key". These metaphors collectively construct dramatic positive change in a decades' old pandemic that continues to affect millions, thereby evoking optimism concerning PrEP. This 'breakthrough' motive is a staple of promissory discourses employed to create positive expectations which foster financial and emotional investment (Brown, 2003).

Articles discussing the results of the iPrEx clinical trial in the US claimed that " $[t]$ his discovery alters the HIV prevention landscape forever,"11 that "Truvada has already made history,",12 and that it was "the most significant breakthrough against the [HIV] virus in a generation." 13 These extracts exemplify the social representation of PrEP as irreversibly altering the set of prevention options currently available and, thus, providing hope. Moreover, PrEP was constructed as superior to the existing prevention methods. The success of PrEP was anchored to Truvada as a treatment option, which served to generalise the benefits of Truvada as treatment to Truvada as PrEP. The importance of PrEP was presented in hyperbolic terms, that is, as "the most significant breakthrough," which elevated the importance of PrEP over and beyond HIV treatment. Although the wide range of treatment options now available to HIV-positive patients, including the one-pill-a-day regimens that obviate the serious problem of low adherence, may plausibly be regarded as a vital "breakthrough" against HIV, PrEP was consistently constructed as surpassing these developments. Reference to the time scale of "a generation" implied that PrEP was the best prevention tool since condoms. Some articles appeared to celebrate the novelty of PrEP, rather than its feasibility per se, as highlighted in the remark that PrEP "was welcomed as a novel approach to HIV prevention." "This signaled a shifting of the HIV debate from treatment to biomedical prevention, as opposed to prevention through condom use.

There is now robust evidence that offering treatment to HIV-positive individuals early, that is, while their CD4 count is still relatively high, has both personal health benefits in the long-term, such as reducing the risk of serious opportunistic infections (SMART Study Group, 2008) and public health benefits because ART significantly reduces the patient's viral load to "undetectable" levels which in turn lowers the risk of onward HIV transmission (Rodger et al., 2014). One article explicitly 
claimed that PrEP "will be better than treatment as prevention." 15 This tapped into the counterargument that most new infections occur through sexual intercourse with a HIV-infected individual who is not virally suppressed because they have not yet initiated ART. Consequently, it was argued that PrEP gives agency to the HIV-negative individual. As in most articles in favor of PrEP, hyperbolic statements were made regarding the contribution of PrEP to mitigating HIV incidence one article noted that "[t]he exciting opportunity that this [PrEP] offers is to make the biggest dent in the epidemic of all time." $" 16$ The contribution of previous campaigns, initiatives and medical developments were attenuated in order to emphasise the importance of PrEP.

The language employed to describe the effect that PrEP may have on HIV prevention further highlighted the optimism surrounding it, as demonstrated in one article that claimed that the results of the PROUD clinical trial "show there is a need for PrEP, and offer hope of reversing the epidemic among men who have sex with men in this country [the UK]." ${ }^{\prime 17}$ Thus, there was an emerging social representation that PrEP might not only prevent HIV but actively reverse and dent it, particularly among gay/bisexual men. Several newspaper headlines read "HIV drug a game changer, says expert," and the metaphor "game-changer" was used in relation to both the clinical trial and PrEP as a prevention tool. ${ }^{18}$ Thus, the metaphors of positive change and the comparisons between PrEP and existing prevention tools contributed to the hope representation.

Consistent with the hope representation of PrEP, many articles focused on the need to make PrEP available to HIV-negative individuals at risk of HIV exposure. For instance, having described PrEP through a journey metaphor as a "massive step in the right direction" in terms of HIV prevention, Professor Sheena McCormack of the Medical Research Council clinical trials unit at University College London, who was also the lead investigator in the PROUD study, was quoted as saying that " $\mathrm{t}]$ he priority now is getting the guys back into the clinic to offer PrEP"19 and, in a sense, send them on a better life journey. Here reference was being made to the realisation among the research team that PrEP provided excellent protection against HIV and that they should offer PrEP to the control group taking a placebo. This laid the rhetorical foundations for arguing in favor of making PrEP widely available, as observed in a commentary in The Guardian that pondered "Can a new drug help to end 30 years of blighted lives?" and affirmatively argued that "[a]n anti-HIV medicine giving peace of mind to gay men in the US should be offered here on the NHS." ${ }^{20}$ This commentary began by highlighting the devastating effect of HIV/AIDS on gay/bisexual communities around the world metaphorically referred to as "blighted lives", thus evoking imagery of injury, pain and devastation, which PrEP could prevent. The commentary proceeded to acknowledge both the potential physical and psychological benefits of PrEP, that is, as an HIV prevention tool but also as one that might facilitate psychological wellbeing among gay/bisexual men who for decades have lived in fear of HIV. This acknowledged the long-standing anxiety surrounding HIV infection among gay/ bisexual men (Shernoff, 2006).

Several articles juxtaposed positive imagery of PrEP and the possibility that it might be offered by the NHS, as suggested in the headlines "Wonder drug' which can reduce HIV risk by $92 \%$ could be offered on the NHS" 21 and "Pressure grows on NHS to make new HIV wonder drug available for those at risk.",2 The metaphor "wonder drug" emphasised the positive imagery of novelty and confidence about the efficacy of PrEP in HIV prevention, differentiating it favourably from other prevention tools. The tentative observations that it "could" be offered on the NHS and that there was "pressure" for it to be offered introduced this possibility in the debate and, in juxtaposition with the positive imagery of PrEP, constructed its endorsement by the NHS as a logical step forward. In some articles, this was presented more forcefully - "HIV pill Truvada that cuts risk of infection among gay men by $86 \%$ needs to be available on the NHS as soon as possible." 23

The social representation of Truvada as a 'wonder drug' can be compared to the metaphor of the 'silver bullet' in other discourses focusing on 'fighting' infectious diseases (Koteyko et al., 2008). Following Breakwell's (2014) model of personalising representations, the salience of the hope representation may plausibly promote awareness and acceptance of PrEP as a highly effective HIV 
prevention method, particularly among gay/bisexual men given the focus on this group in the coverage. However, there was little consideration of its mechanisms (i.e. how PrEP works) or the possible risks associated with it, which may not be conducive to an adequate understanding of the representation. In the following section, we focus on an important aspect of the hope representation, namely the use of militaristic metaphors which are a well-established rhetorical resource when talking about AIDS (Waldby, 1995).

\section{PrEP as a weapon in the battle against HIV/AIDS}

War-related metaphors were employed to render salient and reinforce the hope representation; PrEP was depicted as a weapon in the battle against HIV/AIDS. There was much optimism in reporting on PrEP and its future role in HIV prevention - it was noted that PrEP is expected "to significantly increase the momentum in our fight against the virus." ${ }^{24}$ The context of HIV prevention was constructed metaphorically as a battlefield and HIV as winning the battle before the advent of PrEP. One article described PrEP in the context of the "AIDS battle," ${ }^{25}$ while in others PrEP was identified as "the most significant breakthrough in the battle against the [HIV] virus in a generation" 26 and as a "breakthrough in [the] battle against [the] global scourge." 27 Another article stated that the "US approves pill that guards against HIV [...] potentially offering a new and powerful weapon in the battle against AIDS" ${ }^{28}$ Use of the verb "to guard against" again evoked imagery of military protection, which was further reinforced through use of another war metaphor "weapon." More specifically, PrEP was being characterised not only as a weapon but as a "new and powerful" one. Thus, it provided renewed hope against a virus that has hitherto been characterised as invincible. Despite the constructed power of PrEP, it was represented as gentle to human beings, which is quite different from social representation of ART as causing serious side effects (Bauermeister et al., 2013). In one article, for example, it was claimed that PrEP "offers a safety net" for individuals at risk. ${ }^{29}$

The war framing constructed a very optimistic representation of PrEP, as demonstrated in a commentary piece for The Guardian in which it was referred to as "a powerful addition to the arsenal of weapons against the increasingly besieged HIV virus. ${ }^{30}$ HIV was depicted as being surrounded militarily by external forces compelling its surrender, which referred to the plethora of prevention and treatment options already available (that is, the existing "arsenal of weapons").

Furthermore, it was PrEP in particular that was represented as bringing about a decisive victory in the "battle" against HIV. As discussed above, the role of Truvada as PrEP in HIV prevention was depicted as uniquely important - "Modern anti-retrovirals are phenomenally potent weapons and they could be the key to finally halting the epidemic." 31 As in this extract, there was widespread use of war metaphors, qualified by superlative adjectives (here "phenomenally potent") in order to accentuate the force of PrEP in HIV prevention and, thus, its centrality to "halting the epidemic", another use of a journey metaphor but one focusing on ending rather than starting a journey.

Previous research into representations of infectious disease, such as Foot and Mouth Disease (Nerlich et al., 2002) found that war metaphors dominated discourse and policy, sidelining policies such as vaccination. However, the search for a vaccine has been key in HIV research. In several articles, PrEP was actively anchored to vaccination, which provided a lens for viewing and evaluating the prevention tool. One article, which focused on the results of a European clinical trial, noted that "[p]rotection of 96 to $100 \%$ is better than even the best vaccine." 32 Here, PrEP was being evaluated from the perspective of a vaccine. More specifically, it was depicted as being superior to a vaccine, thereby accentuating the positivity and efficacy of PrEP as a "powerful HIV prevention tool." 33 Furthermore, that article speculated that by encouraging the use of PrEP among "enough inhabitants, the whole viral load of a city can be lowered. That protects everyone - just as vaccination could protect whole communities from smallpox." ${ }^{34}$ PrEP was, thus, anchored to the general notion of vaccination and implied to function in a similar way. It was explicitly anchored to smallpox, which was successfully eradicated due to the introduction of a vaccine. This created an optimistic lens through which to view the potential effects of PrEP on the HIV epidemic. In considering the 
personalisation of the hope representation of PrEP (consisting of its military metaphors) (Breakwell, 2014), it can be argued that the coverage promotes an understanding of PrEP as a highly effective tool capable of eradicating HIV, which in turn is relegated to a victimhood position. Individuals may plausibly activate mental imagery of vaccination in thinking about the prevention tool. However, despite this positive imagery, the hope representation competed with an emerging risk representation.

\section{The risk representation}

There was a competing negative social representation of PrEP which emphasised uncertainties and risks associated with this biomedical option. In contrast to the social representation of breakthrough and hope that focuses on heroes who try to win the fight against a villainous virus, this is primarily a social representation of fear and stigma, where the heroes are recast as villains and the virus emerges as potentially victorious. To encourage acceptance of this representation (Breakwell, 2014), a series of reasons for dismissing PrEP were highlighted in numerous articles, most notably doubts surrounding medication adherence, the effectiveness of PrEP, potential side effects and increased sexual risk-taking.

Several articles pointed to a false sense of hope, which raised questions about the effectiveness of PrEP, thereby challenging the hope representation. One article noted that the approval of PrEP was "given a cautious welcome, but with some experts arguing that it could lead to a false sense of security." 35 The invocation of caution served to challenge the competing hope representation because it introduced the possibility of negativity and risk, which were often understated in pro-PrEP articles. Some articles challenged the growing representation of unanimous endorsement of PrEP in the HIV/AIDS community. This highlighted the uncertainties underpinning PrEP vis-à-vis other prevention tools (e.g. condoms). Several articles reiterated that "PrEP does not work all the time, nor does it prevent sexually transmitted infections like syphilis and gonorrhea." 36 In direct contrast to the hope representation, there was an emerging counter-representation that PrEP was inconsistent in inhibiting HIV infection and completely ineffective in inhibiting other STIs, which reaffirmed the centrality of condoms in HIV prevention. The contestation of positive representations of PrEP constitutes a means of encouraging acceptance of the negative representation of it (Breakwell, 2014).

In addition to questioning the effectiveness of PrEP, many articles pointed to "fears [that] some people would pop a pill and then refuse to worry about contraception,"37 a reference to condom use. The statement that individuals might "pop a pill" suggested that PrEP would be taken blithely with no guarantees about its effectiveness. This specific construction anchored PrEP implicitly to illicit substance abuse, which is the context in which "pop a pill" tends to be employed. This served to anchor potential users of PrEP to drug users, which was particularly important in view of the fact that PrEP would be taken by healthy individuals. Indeed, this was echoed in another article in which PrEP was described as a "party drug" (discussed below). More generally, this risk representation constructed individuals who may benefit from PrEP (e.g. gay/bisexual men) as paradoxically undermining efforts to prevent HIV infection due to their "party" behaviour.

An important strand of uncertainty concerned the sexual risk behaviors of potential PrEP users. In particular, it was argued that PrEP users would cease to use condoms, which evoked the long-standing perception of "condom fatigue" in the gay/bisexual male community (Shernoff, 2006). Condom abandonment was presented as problematic, as preliminary clinical trials suggest that PrEP is not $100 \%$ effective and because it provides protection against HIV infection only and not against other STIs. One article noted that "some critics in the US have warned that the drug could lead to a complacency around condom use and more unsafe sexual behavior" and that there would be an "increase in risk-taking behavior." 38 It claimed that sexual risk-taking might paradoxically "outweigh the prevention effectiveness of Truvada." ${ }^{39}$ These statements served to introduce the (risk) representation of sexual complacency, which undermined a key premise of PrEP that most agree on, namely the need for concurrent use of condoms. 
Pro-PrEP articles cited evidence from clinical trials that PrEP did not decrease condom use in their population sample. However, many articles questioned this emerging evidence. For instance, one article observed that the study "did not prove it unequivocally." 40 Thus, the lack of certainty was presented as cause for scepticism. Another article described the emerging concern among critics that PrEP "will lead to men being more promiscuous or having riskier sex without condoms in the knowledge they were protected from the illness." 41 This tapped into an existing social representation that gay/bisexual men are necessarily promiscuous and prone to engaging in "riskier sex" (Shernoff, 2006). Indeed, this emerging risk representation was reinforced in articles in which generalisations were made about gay/bisexual men's sexual behaviors. One article stated that " $[\mathrm{m}] \mathrm{en}$ generally hate condoms. Their partners usually give in. Almost no one abstains. Precious few stay faithful.",42 Although this article was largely in favor of exploring the use of PrEP among high-risk groups, the social representation of sexual promiscuity among gay/bisexual men contributed to the overarching fear of increased sexual risk-taking. By constructing gay/bisexual men as averse to monogamy and condom use, this article served to represent PrEP as possibly accentuating these "problems" in gay/bisexual men.

Prominent figures in the LGBT and HIV communities were quoted in relation to risk reporting, and strategic expert quoting was employed to construct PrEP as a threat. One article noted that "[s]ome experts remain concerned that PrEP will do more harm than good". There was some media coverage of similar remarks made by prominent gay actor Zachary Quinto. Several articles quoted him as referring to "tremendous complacency" and "laziness" among gay/bisexual men in relation to safer sex practices. ${ }^{44} \mathrm{He}$ reportedly stated that "AIDS has lost the edge of horror it possessed when it swept through the world in the ' $80 \mathrm{~s}$. Today's generation sees it more as something to live with and something to be much less fearful of. And that comes with a sense of, dare I say, laziness." Quinto framed PrEP as an "insurance policy against [...] unprotected non-monogamous sex" which he also called "recreational sex." Quinto, a sexual ingroup member, was himself represented as opposing PrEP on the grounds that it would increase condomless sexual promiscuity, which could severely challenge the effectiveness of PrEP. The metaphor "insurance policy" and the euphemism of "recreational sex" (for promiscuous sex) constructed PrEP as a threatening pathway to increased sexual risk-taking.

Similarly, Weinstein, the president of the AIDS Healthcare Foundation in the US was quoted as referring to Truvada as a "party drug" which would lead to sexual risk-taking. ${ }^{45}$ This extract anchored PrEP to drug use among gay/bisexual men in sexualised settings, commonly referred to as "chemsex." This itself has been linked to rising HIV incidence in these groups (Bourne et al., 2014). Sexual risk-taking among gay/bisexual men was framed in intergroup terms in the UK media's discussions concerning the possible availability of PrEP in the UK, which raised concerns about the potential financial implications for the NHS. While one article questioned the "logic of paying $£ 500$ a month so gay men don't have to wear condoms" in the context of a cash-strapped NHS, another article's headline read: "Why should WE pay for gays to have unsafe sex?" hypothetical scenarios of individuals suffering from cancer and the lack of funding for cancer medication. This positioned PrEP in competition with cancer treatment. These articles constructed rigid intergroup boundaries between the gay/bisexual outgroup, depicted as inherent risk-takers, and the "responsible" (presumably heterosexual) ingroup. PrEP was implicitly positioned as replacing cancer treatment and, thus, as posing a risk not only to HIV-negative individuals but also to cancer patients.

Given these severe criticisms, there was emerging opposition to PrEP. Weinstein was again quoted as referring to the decision by the US Food and Drug Admission (FDA) to approve PrEP as "completely reckless and a move that will ultimately set back years of HIV prevention efforts" and as a move that is "negligence bordering the equivalence of malpractice which will sadly result in new infections, resistance and serious side effects among many, many people." Use of the terms "reckless," "negligence" and "malpractice" by the president of a prominent Los Angeles-based AIDS charity 
gave credibility to the notion that the FDA had hastily and unprofessionally taken a decision that would damage HIV prevention efforts. This cast severe doubt on the trustworthiness of the federal agency and on its ability to protect citizens, thereby decreasing the credibility of the social representations (of PrEP) it endorses and disseminates. Contrary to the hope representation, introduction of the drug was actively represented as harming HIV prevention efforts, thereby bolstering the risk representation. Following Breakwell's (2014) model of personalising representations, it can be argued that these articles promote salience and awareness of the risk representation (drawing on threat and uncertainty) and encourage an understanding of this representation based on complacency, promiscuity and sexual risk-taking.

\section{DISCUSSION}

This article examines emerging social representations of PrEP in the UK press between 2008 and 2015, and develops some speculative hypotheses about possible public responses to them (Breakwell, 2014). The print media make an important contribution to social representations because, collectively, they constitute "a forum for the discourses of others and [are] a speaker in their own right" (Carvalho, 2007, p. 224). They provide space for notions, images and theories to become popularised in the cultures and minds of individuals. The UK newspaper press was ambivalent in its evaluation of PrEP and contributed to both positive and negative social representations of the drug. To this extent, it is appropriate to consider the UK press reporting as highly polarised - as either highly positive or highly negative. Overall, the coverage of PrEP appeared to centre around two major social representations:

- The hope representation accentuates the positive characteristics of PrEP as an HIV prevention method and positions it as superior to existing methods. Through the use of warrelated metaphors, PrEP is positioned as decisively changing the course of HIV prevention. As indicated in Borup et al.'s (2006) work on the sociology of expectations in science and technology, the hope representation can "guide activities, provide structure and legitimation, attract interest and foster investment" in relation to the object of discussion (p. 285-86).

- The risk representation focuses on the risks and uncertainties associated with PrEP, and positions PrEP as a hazard. Through the process of anchoring and objectification, the risks associated with it are rendered familiar and located in a common sense system of meaning (Barnett \& Vasileiou, 2014). As the antithesis of the hope representation, the risk representation is intended to delegitimise PrEP and to discourage interest and investment in it. More generally, the hope representation focuses on taking (individual and collective) responsibility, while the risk representation focuses on attributing (individual and collective) blame.

In order to substantiate the hope representation, articles described PrEP as a superlatively positive development in the campaign against HIV/AIDS through the use of metaphors of positive change and revolution, such as "game changer", "wonder drug" and "revolutionary." Extreme case formulations (Pomerantz, 1986) were employed to emphasise the superiority of this prevention method over and beyond existing methods, especially condoms and treatment as prevention. PrEP was constructed as a novel, distinctive and highly effective prevention method that ought to occupy a preeminent position in HIV prevention. An important rhetorical strategy for constructing the hope representation was undoubtedly the consistent use of war-related metaphors in reporting on how PrEP might halt the HIV/AIDS epidemic. Fundamentally, PrEP was constructed as a weapon - and a very potent one at that - against HIV, which was conversely depicted as besieged in view of this biomedical development. This militaristic strand of the hope representation was further bolstered through the anchoring of PrEP to vaccination - the ultimate "weapon" in previous epidemics that have since been confined to medical history. 
The hope representation hinges upon the triumph of science and the defeat of the virus. HIV was personified as a powerful and evil belligerent that for too long has wreaked devastation on humanity, particularly gay/bisexual men, but which, with the advent of PrEP, would be rendered powerless. This social representation centred around defeating the virus, as has been the case in media reporting on the fight against other emerging infectious diseases such as SARS (Wallis et al., 2005). Crucially, triumph over the virus was constructed as possible through collective efforts. Scientists were positioned as having made significant advances in creating a panacea, drug companies in distributing this panacea, and the NHS was positioned as the final "link" in enabling PrEP to work, that is, by funding provision. The hope representation implicitly assumes that individuals will take responsibility over their sexual health, take their medication regularly and continue to use condoms.

The hope representation competed with a risk representation, which engendered doubts about PrEP by scientific uncertainty as well as risks to human health. This social representation generally questioned the effectiveness of PrEP - not the science but rather the sexual attitudes and behaviours of potential PrEP users. More specifically, the at-risk individual was constructed as inherently irresponsible, lazy, complacent, naïve and incompetent. This was depicted as a consequence of their (sexual) group membership through anchoring to stereotypical images of gay lifestyle (that is, as one characterised by promiscuity and sexual risk-taking). The individual who in principle might benefit from PrEP was simply positioned as unable to contribute to the collective efforts required to prevent HIV.

Furthermore, PrEP was represented as increasing sexual risk-taking among gay/bisexual men (see Hilton et al., 2010 for a similar representation in relation to HPV vaccination among girls). Intergroup dynamics were mobilised primarily on the grounds of sexual orientation. Gay/bisexual men were identified as the sole beneficiaries of PrEP and there was little acknowledgement of the other groups that might benefit from it, such as heterosexual serodiscordant couples, and intravenous drug users. Indeed, PrEP was primarily constructed as a gay/bisexual HIV prevention method. Given this emphasis on gay sexuality in negative press reporting, there was also a moralising discourse that drew heavily on the stigma of gay sexuality as inherently problematic in a heteronormative world. The notion that PrEP might impede other life-saving treatments (e.g. drugs to treat cancer), which, conversely, were positioned as benefiting the general population, was made to appear unacceptable. PrEP was depicted as taking away vital resources from "us" and, thus, as a threat (Stephan \& Stephan, 2000). This is also consistent with the long-standing association of HIV with the "Other" which has long served to distance risk from one's own identity (Washer, 2010). In short, gay/bisexual men's sexual attitudes and behaviours were constructed as endangering themselves but also others, thereby decreasing the effectiveness of PrEP. While the hope representation celebrates the triumph of science and defeat of the virus, the risk representation predicts the defeat of science due to the inertia of the (gay/bisexual) users of PrEP.

The risk representation was further supported through direct efforts to subvert the hope representation. Some articles represented the hope representation as a red herring by constructing a false sense of hope. At a basic level, there was much scepticism about the ability of gay/bisexual men to sustain condom use, although there was no evidence of greater sexual risk-taking in the clinical trials. Furthermore, the metaphor of "popping a pill" constructed a blithe disregard for sexual health. While PrEP was often represented as surpassing condoms, the risk representation firmly reaffirmed the position of condoms as the preeminent prevention tool. In addition to the uncertainty surrounding sustained condom use, some doubt was cast on users' ability to adhere to PrEP which could provide a "false" sense of protection and, thus, increase risk of HIV infection. Incidentally, these concerns have also been mirrored in the findings of existing qualitative interview-based research on PrEP (Tangmunkongvorakul et al., 2013).

Media sociologist Conrad (1997, p. 140) notes that "how we frame a problem often includes what range of solutions we see as possible." There is therefore a need to examine the broader social 
and psychological ramifications of the emerging debates surrounding PrEP in the UK. As Nerlich et al. (2002) have shown with respect to Foot and Mouth Disease, metaphors and narratives have important consequences for how emerging problems are perceived and managed. War and battle metaphors often encourage the perception that this is a battle of good against evil and that good must and will eventually prevail over evil. While the hope and risk representations have a common enemy - HIV, they focus on distinct adversaries and sketch out different pathways for policy and behaviour. The hope representation focuses on the virus as the main adversary and emphasises the collective human struggle against it. The menacing images used to depict PrEP in some articles are downplayed in favour of a united front (Wagner et al., 2002). Conversely, the risk representation appeared to position gay/bisexual sexual risk-takers as the main obstacle in the effectiveness of PrEP and, thus, as endangering themselves and others. The militaristic language added a sense of urgency to deal with these adversaries, either by accepting or rejecting PrEP. The hope representation constructs endorsement of PrEP as necessary and urgent, while the risk representation depicts its endorsement as dangerously irresponsible.

It is important to have a balanced and informed debate about the feasibility of offering PrEP to individuals at high risk of HIV infection. The images, metaphors and social representations that are used in this debate must be carefully selected. If chosen unwisely, they may end up stigmatising the very groups whom PrEP is intended to protect, thereby accentuating the gulf between groups and silencing discourse around sexual health and sexual risk. This may inadvertently contribute to rising HIV incidence. Polarised reporting is unlikely to benefit HIV prevention efforts. While the hope representation alone may create unrealistic expectations about the ability of PrEP to reduce HIV incidence, the risk representation may serve to decrease public and institutional support for PrEP and, due to the stigma it engenders, inhibit access to PrEP among those who could benefit from it.

The print media play an important role in the formation of social representations of PrEP, but representations are also co-constructed, contested or supported through other types of communication - political rhetoric, literature, education, everyday talk, online discussions and so on. It is therefore important that future research engage with discussions concerning PrEP in other social domains, such as on social media platforms and fora, in policy debates and in everyday discourse. Individuals who could benefit from PrEP must take a stance on the emerging social representations - the extent to which they are aware of, understand and accept these representations will be determined by social and psychological factors that require empirical attention in the future (Breakwell, 2014). Indeed, there is a dynamic interplay between representations, identities and personal experiences. Although the data presented in this article exhibit only one small facet of the story regarding social representations of PrEP, we argue that this is an important facet - one that can perform an agendasetting function and introduce representations that are later taken up in societal debates about HIV prevention.

\section{REFERENCES}

Attia, S., Egger, M., Müller, M., Zwahlen, M. \& Low, N. (2009). Sexual transmission of HIV according to viral load and antiretroviral therapy: systematic review and meta-analysis. AIDS, 23(11), 1397-1404.

Baeten, J.M., et al. (2010). Antiretroviral Prophylaxis for HIV Prevention in Heterosexual Men and Women. The New England Journal of Medicine, 367, 399-410

Bauermeister, J.A., Meanley, S., Pingel, E., Solder, J.H. \& Harper, G.W. (2013). PrEP awareness and perceived barriers among single young men who have sex with men. Current HIV Research, 11(7), 520-527. 
Borup, M., Brown, N., Konrad, K. \& van Lente, H. (2006). The sociology of expectations in science and technology. Technology Analysis \& Strategic Management, 18(3/4), 285-98.

Braun, V. \& Clarke, V. (2006). Using thematic analysis in psychology. Qualitative Research in Psychology, 3, 77-101.

Breakwell, G.M. (2014). Identity and social representations. In R. Jaspal and G.M. Breakwell (eds.) Identity process theory: identity, social action and social change (pp. 118-134).

Cambridge: Cambridge University Press.

Brooks, R.A., et al. (2012). Sexual Risk Behaviors and Acceptability of HIV Pre-Exposure Prophylaxis Among HIV-Negative Gay and Bisexual Men in Serodiscordant Relationships: A Mixed Methods Study. AIDS Patient Care \& STDs, 26(2 ), 87-94.

Brown, N. (2003). Hope against hype-accountability in biopasts, presents and futures. Science Studies, 16(2), 3-21.

Carvalho, A. (2007). Ideological cultures and media discourses on scientific knowledge: Rereading news on climate change. Public Understanding of Science, 16, 223-224.

Conrad, P. (1997). Public eyes and private genes: Historical frames, news constructions, and social problems. Social Problems, 44, 139-154.

Deacon, D. (2007). Yesterday's papers and today's technology: digital newspaper archives and 'push button' content analysis. European Journal of Communication, 22(1), 5-25.

Eicher, V. \& Bangerter, A. (2015). Social representations of infectious diseases. Cambridge: Cambridge University Press.

Entman, R.M. (1989). How the media affect what people think: an information processing approach. The Journal of Politics, 51(2), 347-70.

Flash, C., et al. (2014). Two years of Truvada for pre-exposure prophylaxis utilization in the US. Journal of the International AIDS Society, 17(3), 19730.

Fogarty, L., Roter, D., Larson, S., Burke, J., Gillespie, J. \& Levy, R. (2001). Patient adherence to HIV medication regimens: a review of published and abstract reports. Patient Education \& Counseling, 46(2), 93-108.

Golden, M.R., Stekler, J., Hughes, J.P. \& Wood, R.W. (2008) HIV Serosorting in Men Who Have Sex With Men: Is It Safe? Journal of Acquires Immune Deficiency Syndromes, 49(2), 212-18.

Grant, R.M. et al. (2010). Preexposure Chemoprophylaxis for HIV Prevention in Men Who Have Sex with Men. The New England Journal of Medicine, 363, 2587-2599.

Herek, G. M. (1990). Illness, stigma, and AIDS. In P. T. Costa, Jr. \& G. R. VandenBos (Eds.), Psychological aspects of serious illness: Chronic conditions, fatal diseases, and clinical care (pp. 103-150). Washington, DC: American Psychological Association. 
Jaspal, R., Nerlich, B. \& Koteyko, N. (2013). Contesting science by appealing to its norms: Readers discuss climate science in The Daily Mail. Science Communication, 35(3), 383-410.

Koteyko, N., Nerlich, B., Crawford, P. and N. Wright (2008) “'Not rocket science' or 'no silver bullet'? Media and government discourses about MRSA and cleanliness". Applied Linguistics, 29, 223-243.

Lacor, P. (2012). Preparing for PrEP. The European Journal of Contraception and Reproductive Health Care, 17, 482-486.

Lakoff, G. \& Johnson, M. (1980). Metaphors We Live By. Chicago: University of Chicago Press.

McCormack et al. (2016). Pre-exposure prophylaxis to prevent the acquisition of HIV-1 infection (PROUD): effectiveness results from the pilot phase of a pragmatic open-label randomised trial. The Lancet, 387(10013), 53-60.

Molina J. M. et al. (2015). On demand PrEP with oral TDF-FTC in MSM: results of the ANRS Ipergay trial. CROI 2015. 2015 Conference on Retroviruses and Opportunistic Infections (CROI 2015), 23-26 February 2015, Seattle, Washington, USA. Oral late breaker abstract 23LB.

Moscovici, S. (1988) Notes towards a description of social representations. European Journal of Social Psychology 18: 211-250.

Nerlich, B., Hamilton, C.A. \& Rowe, V. (2002). Conceptualising foot and mouth disease: the socio-cultural role of metaphors, frames and narratives. Metaphorik.de,2. http://www.metaphorik.de/02/nerlich.pdf

Pomerantz, A. (1986). Extreme case formulations: a way of legitimizing claims. Human Studies, 9, 219-229.

Public Health England (2015). HIV in the UK-Situation Report 2015: Incidence, prevalence and prevention. London: Public Health England.

https://www.gov.uk/government/uploads/system/uploads/attachment_data/file/477702/HIV in the UK 2015 report.pdf

Reese, S., Gandy, O. \& Grant, A. (Eds.) (2003). Framing Public Life: Perspectives on media and our understanding of the social world. Mahwah, NJ: Erlbaum.

Rodger A et al. (2014). HIV transmission risk through condomless sex if HIV+ partner on suppressive ART: PARTNER study. 21st Conference on Retroviruses and Opportunistic Infections, Boston, abstract 153LB.

Rose, D., Efraim, D., Gervais, M., Joffe, H., Jovchelovitch \& Morant, N. (1995).

Questioning consensus in social representations theory. Papers on Social Representations, $4(2), 1-6$.

Shernoff, M. (2006). Without condoms: unprotected sex, gay men \& barebacking. London: Routledge. 
SMART Study Group (2008). Inferior clinical outcome of the CD4+ cell count-guided antiretroviral treatment interruption strategy in the SMART study: role of CD4+ Cell counts and HIV RNA levels during follow-up. Journal of Infectious Diseases, 197(8), 1145-55.

Sontag, S. (1978). Illness as metaphor. New York: Farrar, Straus \& Giroux.

Sontag, S. (1989). AIDS and its metaphors. New York: Farrar, Straus \& Giroux.

Spieldenner, A. (in press). PrEP whores and HIV prevention: the queer communication of HIV pre-exposure prophylaxis. Journal of Homosexuality.

Stephan, W.G. \& Stephan, C.W. (2000). An integrated threat theory of prejudice. In S. Oskamp (Ed.), Reducing prejudice and discrimination (pp. 23-46). Mahwah: Erlbaum.

Tangmunkongvorakul, A., et al. (2012). Facilitators and barriers to medication adherence in an HIV prevention study among men who have sex with men in the iPrEx study in Chiang Mai, Thailand. AIDS Care, 25(8), 961-967.

The TenoRes Study Group (2016). Global epidemiology of drug resistance after failure of WHO recommended first-line regimens for adult HIV-1 infection: a multicentre retrospective cohort study. The Lancet, Online First, DOI: http://dx.doi.org/10.1016/S1473-

3099(15)00536-8

Wagner, W., Kronberger, N. \& Seifert, F. (2002). Collective symbolic coping with new technology: Knowledge, images and public discourse. British Journal of Social Psychology 41: 323-343.

Waldby, C. (1995). Body wars, body victories: Aids and homosexuality in immunological discourse. Science as Culture, 5(2), 181-198.

Wallis, P. \& Nerlich, B. (2005). Disease metaphors in new epidemics: the UK media framing of the 2003 SARS epidemic. Social Sciences \& Medicine, 60(11), 2629-39.

Washer, P. (2010). Emerging infectious diseases and society. Basingstoke: Palgrave.

\footnotetext{
${ }^{1}$ Truvada ${ }^{\circledR}$ consists of two reverse transcriptase inhibitors, tenofovir disoproxil fumarate and emtricitabine, both of which are first-line drugs currently used to treat HIV infection.

${ }^{2}$ http://www.unaids.org/en/resources/campaigns/HowAIDSchangedeverything/factsheet

${ }^{3}$ Centre for Disease Control http://www.cdc.gov/hiv/basics/prep.html

${ }^{4} \mathrm{http} / /$ www.usatoday.com/story/news/nation/2014/04/06/gay-men-divided-over-use-of-hiv-preventiondrug/7390879/

${ }^{5}$ http://www.businesswire.com/news/home/20150817006348/en/AHF-Highlights-\%E2\%80\%9CReachingCommon-Ground-PrEP\%E2\%80\%9D-National\#.VdmJCXgUvEZ

${ }^{6} \mathrm{http}: / /$ www.thehastingscenter.org/Bioethicsforum/Post.aspx?id=6777\&blogid $=140$

${ }^{7}$ https://www.england.nhs.uk/2016/03/prep/; http://www.prepaccess.org.uk/

${ }^{8} \mathrm{PEP}$ is prescribed only to patients who are likely to have been at high risk of HIV exposure (i.e. following condomless anal sex with an HIV-positive individual with a detectable viral load). A typical PEP regimen consists of Truvada (two reverse transcriptase inhibitors) and Raltegravir (an integrase inhibitor), which must be started within 72 hours of the possible exposure and continued for 30 days.

${ }^{9}$ PrEP Facts: Rethinking HIV Prevention Facebook Group https://www.facebook.com/groups/PrEPFacts FDA Guidance for Industry
} 
http://www.fda.gov/downloads/drugs/guidancecomplianceregulatoryinformation/guidances/ucm401087.pdf

${ }^{10}$ Nexis $^{\circledR}{ }^{1}$ is a convenient source for the analysis of the traditional print media, although there are some limitations (Deacon, 2007). A significant drawback is that this data source is frequently reconfigured, leading to slightly different results obtained when consulting Nexis ${ }^{\circledR}$ at different dates. Nevertheless, it provides a sufficiently large corpus so as to identify general social representations prevalent at a given point.

${ }^{11}$ The Scotsman,24/11/2010

${ }^{12}$ Metro, 15/10/2012

${ }^{13}$ Independent, $16 / 10 / 2014$

${ }^{14}$ Telegraph,24/02/2015

${ }^{15}$ Independent, 16/10/2014

${ }^{16}$ Independent, 17/10/2014

${ }^{17}$ Belfast Telegraph,24/02/2015

${ }^{18}$ Irish Examiner, 25/02/2015

${ }^{19}$ The Times, 17/10/2014

${ }^{20}$ The Guardian,29/09/2014

${ }^{21}$ MailOnline, 17/10/2014

${ }^{22}$ MailOnline, 17/05/2014

${ }^{23}$ Belfast Telegraph,24/02/2015

${ }^{24}$ Belfast Telegraph,24/02/2015

${ }^{25}$ MailOnline, 11/07/2014

${ }^{26}$ Independent, 17/10/2014

${ }^{27}$ Independent, 25/02/2015

${ }^{28}$ The Guardian, 17/07/2012

${ }^{29}$ The Guardian,29/09/2014

${ }^{30}$ The Guardian,29/09/2014

${ }^{31}$ Daily Mirror,8/09/2014

${ }^{32}$ Daily Mirror,8/09/2014

${ }^{33}$ MailOnline, 17/10/2014

${ }^{34}$ Daily Mirror,8/09/2014

${ }^{35}$ Metro, 15/10/2012

${ }^{36}$ Telegraph,24/02/2015

${ }^{37}$ Metro, $15 / 10 / 2012$

${ }^{38}$ Independent, $16 / 05 / 2014$

${ }^{39}$ Metro, 15/10/2012

${ }^{40}$ Independent, $25 / 02 / 2015$

${ }^{41}$ Daily Mail,26/02/2015

${ }^{42}$ Daily Mirror,8/09/2014

${ }^{43}$ Independent, 16/05/2014

${ }^{44}$ Independent, $15 / 11 / 2014$

${ }^{45}$ Independent, $16 / 05 / 2014$

${ }^{46}$ MailOnline, 28/02/2015 